\title{
Mixed-potential based direct catalyst conversion sensor: Independency of the sensor response from oxygen, electrode material, and from the type of analyte
}

\author{
Thomas Ritter, Gunter Hagen, Ralf Moos \\ Department of Functional Materials, Bayreuth Engine Research Center, University of Bayreuth, \\ Germany
}

\begin{abstract}
:
On-board diagnostics (OBD), which ensures the functionality of exhaust gas aftertreatment systems of combustion engines, needs reliable gas sensors. Therefore, we investigate a sensor that is able to determine directly catalyst conversion (between 0 and 100\%). A mixed-potential is formed at two similar electrodes connected to different gas atmosphere, e.g. up- and downstream of a catalyst. Here, it is simulated by gas mixtures containing an analyte, e.g. a hydrocarbon. These mixtures can be adjusted so that different conversion of the catalyst is simulated. Identical analyte concentration indicate zero conversion and zero sensor response. The higher the catalyst activity is, the lower is the analyte concentration downstream of the catalyst and the mixed-potential at the second electrode increases. Theoretically, the sensor signal is only dependent on the conversion of the catalyst but on the analyte concentration of the feed gas. In this work, we verify these considerations for the first time also for the case of analyte mixtures, Au-electrodes, or varying oxygen contents.
\end{abstract}

Key words: solid state mixed-potential sensor, direct conversion sensor, on-board diagnostics (OBD), hydrocarbon sensor, high temperature co-fired ceramics (HTCC)

\section{Sensing principle}

It was recently shown that the conversion of a catalyst can be determined by an electrochemical comparison of the two gas mixtures up- and downstream of a catalyst. Hereby, the non-Nernstian behavior is utilized. Mixed-potentials are formed at two (similar) electrodes in different gas atmospheres. According to the difference in the analyte concentrations of both atmospheres, different half-cell potentials form. As the electrodes are connected by an ion-conductor (YSZ), a sensor voltage depending on the concentration gradient acts as the sensor response. Theoretical considerations (for details see [1]), show that the sensor signal $U_{\mathrm{s}}$, which is the measured voltage between the mixed potentials formed at the reference electrode (RE) and the working electrode (WE), depends only on catalyst conversion $\xi$ and not on the feed gas concentration:

$U_{S}=$ const. $\cdot \ln (1-\xi)$

We already presented a planar self-heated sensor setup in high-temperature co-firing ceramics technology, providing sufficient high temperature for sensing (up to at least $550{ }^{\circ} \mathrm{C}$ ) and - simultaneously - hermetic sealing of the two gas mixtures, as described in [2]. With such devices, the theoretical consideration were verified, using $\mathrm{Pt}$ thick-films as functional electrodes and propene $\left(\mathrm{C}_{3} \mathrm{H}_{6}\right)$ as test gas.

\section{Setup}

Now, the sensor electrodes are designed as a mesh (Fig. 1a). This geometry provides a minimum of Pt amount while the electrode area and triple phase boundaries are still sufficient. This ensures an optimal mixed-potential sensor performance, since an excess of Pt could cause high catalytic activity, which equilibrates the gas mixtures and inhibits the formed mixed potentials, as it was shown in [1]. To simulate the atmospheres up- and down-stream of a catalyst, each electrode is exposed to a synthetic gas mixture composed by mass flow controllers. Here, the reference side of the setup (RE, gas in- / outlet "1") was purged with a constant analyte concentration. The working electrode (WE) faces the atmosphere where the analyte concentration decreases stepwise (gas in- / outlet "2") to simulate increasing catalyst conversion. The sensor signals now are plotted over the calculated conversion value $\xi$ coming from the relation between the analytes concentrations " 1 " and " 2 ". A scheme of the sensor setup is given in Fig. 1b).

In contrast to our former work, the herepresented experiments were conducted with 
varying oxygen contents, electrode materials or gas mixtures that served as the analyte.

\section{Results}

Firstly, the question was addressed whether the principle can be transferred to other gases than only to $\mathrm{C}_{3} \mathrm{H}_{6}$. A mixture containing propene, ethane, ethene and acetylene (each contribute with $25 \%$ to the total hydrocarbon concentration $\mathrm{THC}$ ) was dosed with varying feed gas concentration. As it can be seen in Fig. 2, the theory fits excellent with the measured values regarding the line calculated with an offset corrected equation (1). In a second experiment, the oxygen content in the feed gas (meaning both, the reference ("1") and the test gas atmosphere "2") were varied. Acc. to (1), the oxygen content does not influence the response. This test was conducted using gold electrodes on both sides and again propene as the analyte. As shown in Fig. 3, no considerable influence on the correlation between response and conversion can be seen.

a)

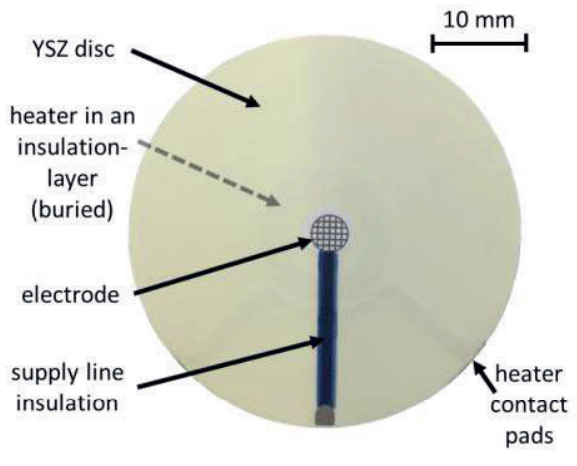

In addition, the use of Au electrodes demonstrates the universal validity of the theory. Although the signal is a little lower, it could be shown that also $\mathrm{Au}$ as electrode material leads to stable sensor responses. Future work will focus on using electrode materials that are selective to a certain analyte to exclude cross effects in real exhaust gas mixtures. Preliminary data provide evidence that even humidity barely affects the sensor signal.

\section{References}

[1] T. Ritter, G. Hagen, J. Lattus, R. Moos: Solid state mixed-potential sensors as direct conversion sensors for automotive catalysts, Sensors and Actuators B: Chemical 255, 30253032 (2018), doi: 10.1016/j.snb.2017.09.126

[2] T. Ritter, G. Hagen, J. Kita, S. Wiegärtner, F. Schubert, R. Moos: Self-heated HTCC-based ceramic disc for mixed potential sensors and for direct conversion sensors for automotive catalysts, Sensors and Actuators B: Chemical 248, 793-802 (2017), doi: 10.1016/j.snb.2016.11.079

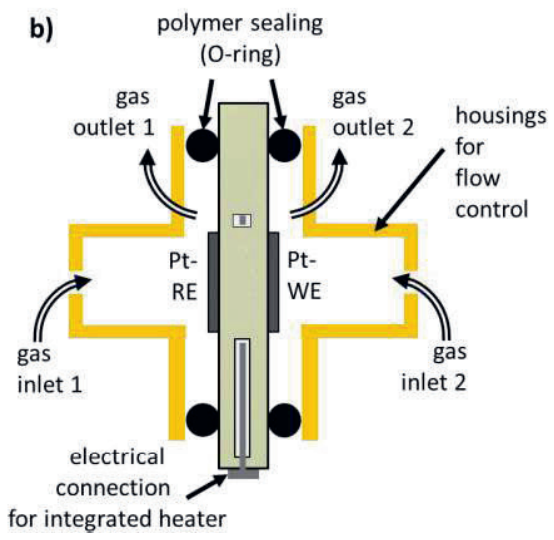

Fig.1. a) Image of the front side of a sensor device as it was described in [1], while here the electrode is designed as a Pt mesh that ensures excellent mixed-potential sensor performance. b) Scheme of the cross section of the sensor setup as it was used for the experiments.

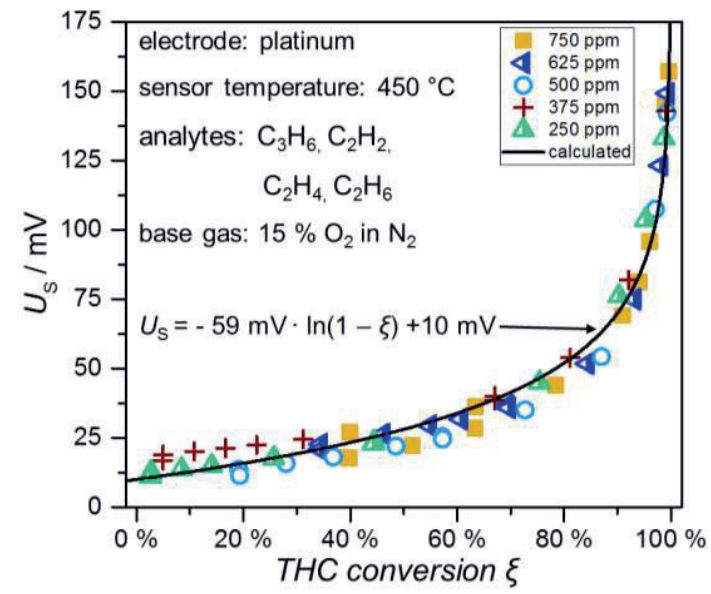

Fig. 2. Sensor response over total hydrocarbon conversion when varying feed gas concentrations of a mixture containing propene, ethane, ethylene and acetylene were dosed. The calculated line was fitted to meet the measured values with an offset corrected equation (1).

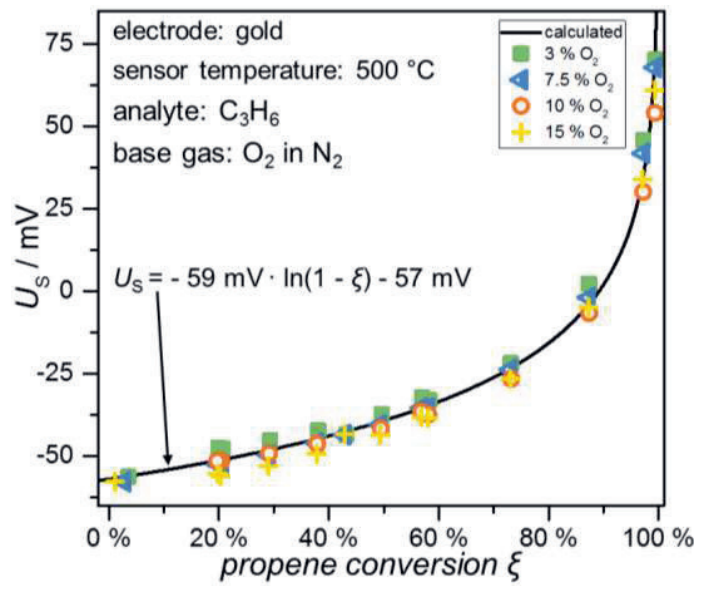

Fig. 3. Sensor signal over propene conversion on dosing both $250 \mathrm{ppm}$ and $500 \mathrm{ppm}$ propene containing $\mathrm{N}_{2}$ gas mixtures with a varying oxygen content. The calculated line was fitted to meet the measured values with an offset corrected equation (1). 